\section{NOVA TELLVS}

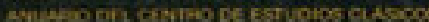

Nova Tellus

ISSN: 0185-3058

novatelu@servidor.unam.mx

Centro de Estudios Clásicos

México

Taboada, Hernán G. H.

Los clásicos entre el vulgo latinoamericano

Nova Tellus, vol. 30, núm. 2, 2012, pp. 205-219

Centro de Estudios Clásicos

Distrito Federal, México

Disponible en: http://www.redalyc.org/articulo.oa?id=59128313008

- Cómo citar el artículo

- Número completo

- Más información del artículo

- Página de la revista en redalyc.org

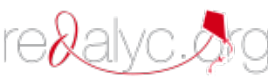

Sistema de Información Científica

Red de Revistas Científicas de América Latina, el Caribe, España y Portugal

Proyecto académico sin fines de lucro, desarrollado bajo la iniciativa de acceso abierto 


\title{
Los clásicos entre el vulgo latinoamericano
}

The Classics among the Latin American Common People

\author{
Hernán G. H. TABOADA \\ Universidad Nacional Autónoma de México \\ haroldo@unam.mx
}

\begin{abstract}
RESUMEN: Entre las estrategias de legitimación de las clases dominantes criollas ha figurado la apelación a los clásicos grecorromanos como basamento de la cultura latinoamericana, lo cual ha resultado en una serie de discursos oficiales o elitistas muy hieráticos y poco convincentes, traducidos de la Europa burguesa. Pero también ha llevado a una reelaboración de dicha herencia clásica por parte de grupos plebeyos que en realidad responden a otras tradiciones culturales, como puede verse en la onomástica, la poesía popular y otros campos. Las masas latinoamericanas han sabido ver más allá de las elites criollas y han adivinado en su reelaboración una imagen de la antigüedad más verdadera que la versión hierática ofrecida por la cultura oficial.
\end{abstract}

ABSTRACT: The reference to Classics as the basis of Latin American culture has been one of the strategies of legitimacy of the Creole classes. The result was a range of very hieratic and unconvincing official or elite discourses, translated from bourgeois Europe. Another consecuence of the said propaganda was the acceptance of Classical legacy by plebeian groups that, in fact, belonged to different cultural traditions (as can be seen in personal names, popular poetry and other fields). However, it seems that Latin American masses were able to see beyond the Creole elites, and guessing in their remake an image of Antiquity truer than the one offered by the official culture.

PALABRAS CLAVE: cultura latinoamericana, tradición clásica, cultura popular.

KEY WORDS: Latin American culture, classical Tradition, popular culture.

RECIBIDO: 23 de mayo de 2012 - ACEPTADO: 16 de enero de 2013.

¿De dónde íbamos a resultar helenos nosotros, zambitopos vocingleros, cholitos hirsutos? ${ }^{1}$

Helenizando a la chusma

Si bien en la cultura criolla el dominio del latín fue y sigue siendo minoritario, por no hablar del griego, los clásicos grecolatinos y su mundo constituyen una parte central del imaginario latinoamericano. Aunque se ha explicado aludiendo a nuestras lejanas raíces, la razón principal

${ }^{1}$ Sánchez 1968. 
es la insistencia en el tema por parte de los grupos dominantes, que han llegado a colocar a tales clásicos como el basamento de su herencia e incluso han logrado extender su prestigio hasta las capas más bajas de la sociedad, que también terminan creyéndose descendientes de Grecia y Roma o, por lo menos, reproduciendo un eco alejado de las mismas.

La prédica en este sentido está enlazada con los símbolos mismos de las repúblicas criollas. Los escudos de nuestros países los incluyen: columnas dóricas, fasces romanas, laureles, cornucopias, matronas. Si en los comienzos de la independencia el escudo de la Gran Colombia ostentaba a una mujer india, ésta luego fue sustituida por la Libertad romana. ${ }^{2}$ Los himnos nacionales, diariamente entonados en escuelas o medios de comunicación, son igualmente pródigos en las referencias: “cual Rómulo y Remo", "cual Febo entre nubes" (Paraguay), "la lanza de Marte / de Bruto el puñal" (Uruguay), "Marte mismo parece animar" (Argentina), "centauros indomables / Termópilas brotando, constelación de cíclopes" (Colombia). ${ }^{3}$ La toponimia misma fue clasicizada: dejemos el intento de Francisco Antonio Ulloa, quien en 1814 propuso cambiar el nombre de Abejorral, llamándola Mesenia; de Baos, para convertirla en Larisa; mientras Guarne sería Élida; Urrao, Olimpia; Canoas, Canope y Angostura, Amicla. ${ }^{4} \mathrm{El}$ aprovechamiento del sonsonete Canoas-Canope en esta propuesta fracasada nos recuerda otra que sí triunfó, la de la Esparza costarricense transformada en Esparta. Y sin sonsonete, la toponimia criolla de las décadas siguientes a la independencia abrevó generosamente en el mundo mediterráneo, de manera que hoy vemos figurar en nuestros mapas a Atenas, Corinto, Palmira, Nueva Alejandría, Esparta, Tesalia, Calcedonia, Salamina, Gorgona y Pireo.

Y cuando las repúblicas se organizaron y enriquecieron, el paisaje urbano se llenó de edificios y estatuas griegas y romanas. Junto a parlamentos, mausoleos y casas de gobierno neoclásicas, la ciudad de México puede ostentar una Diana Cazadora, una Victoria de Samotracia copiada de la griega y una Cibeles copiada de la madrileña; mientras hay una Minerva en una zona residencial de Guadalajara, un Ícaro y Dédalo en Santiago de Chile, una Fuente de Venus en Buenos Aires, y muchas otras más desconocidas o peor ejecutadas en cementerios o casas particulares.

\footnotetext{
${ }^{2}$ Guerrero 1997.

${ }^{3}$ Las recoge Gutiérrez Estévez 2004.

${ }^{4}$ Vilanova 1998.
} 
Los héroes nacionales asumieron actitudes e indumentaria clásica, y cuando no, se quiso que a la fuerza lo hicieran: en ocasión del develamiento en Montevideo de una estatua dedicada a la Victoria, se dijo de ella que tenía facciones griegas y que llevaba una sobretúnica, una corona, unas boleadoras y una lanza tacuara. En realidad, las facciones no son griegas, sino indias; la corona bien puede ser una vincha con plumas; la sobretúnica, un vil poncho, y la rama de laurel, un árbol autóctono. ${ }^{5}$

La intención de diseminar tanto perfil mitológico y heroico la expresó sin querer un dirigente argentino, quien, al abogar por una educación que estuviera regida, más que por estudios formales, por el ejemplo de clases superiores, traía a colación las escaleras de mármol y la Diana que adornaban el Jockey Club de Buenos Aires, ante la cual "el indio más guarango quedaba vencido y dominado". ${ }^{6}$ En efecto, el objetivo final de los clasicistas criollos era el de civilizar al paisaje y a la sociedad americana, a la chusma misma: la cita latina fue compañera inseparable de la represión y el chicote en nuestra historia. ${ }^{7}$

Ilusionándose con ello, llegaron a retratar a nuestros rústicos con pinceles bucólicos. Primero fue en las artes visuales, donde por falta de otros modelos, los grabadores o pintores europeos habían asignado a mexicas o guaraníes cuerpos y poses helenas, estilo que continuó en la estatuaria cívica de nuestras capitales. Después en la literatura, donde abundaron las descripciones arcádicas de América, con ejemplos que van desde la Rusticatio Mexicana (1781), poema evocativo en latín del guatemalteco Rafael Landívar, hasta la Égloga trágica (1916), novela del ecuatoriano Gonzalo Zaldumbide, o los Cantos de Cifar y del Mar Dulce (1969-1979), del nicaragüense José Antonio Cuadra. Incluso el ensayo pretendió hacer de nosotros unos herederos de aquella Hélade luminosa que los criollos en turno habían copiado de revistas francesas: Leopoldo Lugones y Ricardo Rojas practicaron la operación con los gauchos argentinos, en los que percibían vocabulario, actitudes, atuendos y

${ }^{5}$ Demasi 2005, p. 1040.

${ }^{6}$ Devoto 2002, pp. 90-91.

7 'El 'gendarme necesario' de Laureano Vallenilla Lanz constituye una forma criolla del Dux veneciano o del tirano griego", dice en un obiter dictum Sánchez 1968, p. 22. Fuera de él sólo conozco un estudio que rastrea la función disciplinadora de los clásicos en América Latina, en Colombia más precisamente, desde el conquistador latinista Gonzalo Jiménez de Quesada hasta el conservador presidente Manuel Antonio Caro, ambos expertos en el uso simultáneo de la cita ciceroniana y el palo; véase Soto Posada 2007. 
nombres de ese origen. Tan convincente resultaba la argumentación que pudo publicarse como el libro de Lonko Kilapán, El origen griego de los araucanos, donde se estudian unas largas migraciones desde Esparta hasta los valles chilenos y una de las pruebas que se muestra es la comparación entre una estatua espartana y otra de Caupolicán. ${ }^{8}$

No nos quedamos en esfuerzos puramente intelectuales. La retórica era capaz de entonar discursos de este tenor:

Vivimos una democracia digna de los tiempos del ágora. Ésta es la ciudad de Platea, está con nosotros Arístides el justo; venimos a celebrar una gran Eleuteria. En esta pira sagrada inmolemos eternamente nuestros corazones para que no se extinga. Costa Rica entera sea la vestal virtuosa: pura de pensamiento; limpia de obra; afanosa de acción; pletórica de cultura, pero siempre dispuesta al sacrificio. ${ }^{9}$

Periódicamente, la educación pretende imponer el latín en los planes de estudio, y hasta hubo intentos más serios con la difusión de los concursos de belleza a principios del siglo Xx, que en Brasil expresaron propósitos eugenésicos: su anuncio entre columnas jónicas buscaba la elección de una "Miss Brasil" con los rasgos clásicos de una estatua griega. No faltaron en México esfuerzos similares para mejorar la raza. ${ }^{10}$

\section{La chusma se convierte}

La insistencia llevó a que gentes de muy distinta tradición se sumergieran en el estudio de aquellos clásicos prestigiosos. En su defensa de las lenguas antiguas, fray Manuel de San Juan Crisóstomo Nájera alegaba en 1843 que aprender griego le era posible incluso a los mexicanos, y aportaba el ejemplo del Cayetano Cabrera, descendiente de negros e indios pero capaz de pluma ciceroniana. ${ }^{11}$ Un poeta popular como el mulato cubano Plácido realizó pastiches de ninfas y filósofos, "como lacayo que se viste con las ropas de su señor", según un irónico Marcelino Menéndez Pelayo. ${ }^{12}$

\footnotetext{
${ }^{8}$ Kilapán 1974.

${ }^{9}$ Urbina Gaitán 1929.

${ }^{10}$ Stepan 1991, p. 134; Besse 2005.

${ }^{11}$ Osorio Romero 1986.

12 Menéndez Pelayo 1911, tomo 1, p. 262.
} 
El brasileño Machado de Assis, cuando fue llamado mulato por los estadounidenses, se definió como heleno, tanto como el otro mulato Tobías Barreto, "griego, pequeño y fuerte" (1862). ${ }^{13} \mathrm{O}$ el mexicano Enrique Gómez Robelo, "un indio antiguo, cuya melancolía se disipaba al acordarse de que también era griego de la decadencia". ${ }^{4}$

Y más abajo aún. Entre los círculos llamados “Ateneo" que pulularon, algunos reunían al bajo pueblo de mestizos o de negros. Entre la ironía y la aprobación, el español Luis Araquistain citaba el caso de aquel negro de Puerto Rico que "perorando en un mitin decía golpeándose las venas de su brazo bituminoso: 'Nosotros, los de la raza latina', variante del 'Civis sum Romanus"”. ${ }^{15}$ Un caso más de la asunción de este gentilicio criollo por individuos que sólo tienen remota idea de lo que significa. En sus recorridos por la costa atlántica colombiana en 1859, el geógrafo francés Elisée Reclus fue interrogado por unos pescadores mestizos de La Ciénaga, entre el hedor del pescado, sobre Europa, Francia, Madrid; de pronto

le plus jeune des pêcheurs, celui qui m'écoutait avec le plus d'intérêt, avait, je ne sais où, entendu parler d'Athènes. Il m'interrompait souvent. "On dit qu'il y a de bien beaux temples à Athènes! On sculpte de belles statues à Athènes! L'université d'Athènes est la plus célèbre du monde entier, n'est-ce pas? Aucune langue n'est aussi belle que le latin d'Athènes?" Chose étrange que cet écho lointain de la Grèce sur les dunes de l'Atlantide! La gloire de Phidias et de Périclès a mis deux mille ans à franchir les mers, et maintenant des pêcheurs américains s'en entretiennent, comme si cette gloire était encore la plus rayonnante de l'ancien monde! ${ }^{16}$

La afición perduraba cuando Mariano Picón Salas conoció a unas gentes de Maracaibo que, "para que no los apodaran fenicios, tenían sus poetas propios y sus mitos indígenas regionales [...] el neoclasicismo de sus maestros de escuela exigía que junto a la rumorosa Calle del Comercio existiera la Calle de las Ciencias y que cualquier rapaz se nombrara Aristóteles o Sócrates". Por otra parte, en los Ateneos del Zulia, la mitología

\footnotetext{
13 Véase la introducción a Lima Barreto 1978.

${ }^{14}$ Valle 1952, p. 260.

${ }^{15}$ Luis Araquistain (1930), citado en Fornet 2000, p. 198.

16 Reclus 1859.
} 
clásica era tan familiar como la nativa. ${ }^{17}$ En Cochabamba imperaba un "conocimiento superficial de la historia, y son aficionados a evocar épocas remotas, citar nombres de héroes griegos o romanos y narrar con sus detalles los culminantes episodios de la Revolución francesa", así como la historia patria. ${ }^{18}$ En la ciudad de la provincia argentina que bajo el nombre de "Pago Chico" nos describió Roberto Payró, nadie, ni siquiera el cura, es capaz de traducir correctamente un corto latinajo; sin embargo, uno de los notables tiene en su jardín un Apolo, "estatua de yeso pintado imitando mármol veteado, que tanto podía representar a un tullido", y el tendajón de un inmigrante italiano pretende un aura clásica: "sapatería e spacio de bevida di Romulo e Remo di Giuseppe Cardinali". ${ }^{19}$ En Canaima (1935), Rómulo Gallegos recordaba la figura de aquel tendero de la frontera amazónica que consideraba la mitología clásica como el repositorio simbólico de "todo cuanto pueda ocurrir en la vida de una persona o una colectividad". ${ }^{20}$

De esta influencia popular da cuenta en algún lugar el mexicano Martín Luis Guzmán nada menos que en los días afanosos de la Revolución mexicana:

Caí en la cuenta que mi evocación de Roma en el primer contacto con el constitucionalismo revolucionario habanero respondía a una presencia más profunda de lo que parecía a primera vista. El pensamiento romano, en efecto, traía muy preocupado en esos días a González Blanco y Zubarán. En las polémicas que uno y otro sostenían con periódicos y escritores vendidos a la causa de Victoriano Huerta, los argumentos máximos de ambas partes no se referían por lo común a la historia de México, sino a la de Roma, y se fundaban en sentencias y máximas sacadas de los oradores, historiadores y políticos del siglo de Augusto. Se combatía al usurpador en nombre de Mario y Sila; se le defendía con apoyo en la rivalidad de Pompeyo y César; lo decisivo en cada réplica eran las citas de Cicerón, los pasajes de Tito Livio. Todo ello latinidad barata, latinidad de ediciones Sempere, mas no desprovisto de brío y linaje.

Entusiasmo que contagió al general José Isabel Robles, al que Guzmán una vez encontró leyendo a Plutarco en un volumen que había

\footnotetext{
${ }^{17}$ Picón Salas 1949, p. 45.

${ }^{18}$ Arguedas 1977, p. 87.

${ }^{19}$ Payró 1969, pp. 14, 24, 111.

${ }^{20}$ Gallegos 1935, p. 103.
} 
saqueado de una residencia: "iQuién hubiera vivido en esos tiempos de Grecia y Roma!”, exclamaba, para lanzar contra los políticos: “¡De todos estos discurseadores no se saca un Demóstenes!". ${ }^{21}$

El acercamiento se tradujo en un rasgo que varios extranjeros notaron: la frecuencia de alusiones clásicas en la onomástica latinoamericana. Quizá el origen lejano está en la costumbre de los dueños de esclavos, que las colonias españolas compartieron con las colonias inglesas y francesas: “ ¡Rojana! ¡Estativa!, ¡Olimpia!, ¡Aspasia!, ¡Timotea! - y otras tantas negrillas esclavas de quien el señor Estaca había hecho una traducción andante de Quinto Curcio, dejaron los míseros lechos en que dormían y acudieron espantadas a los gritos del amo", en la novelada Lima de Vicente Fidel López. ${ }^{22}$ Luego intervinieron factores de moda, el anticlericalismo o la creencia anarquista en un papel significativo de los grandes hombres. En el mundo del tango tenemos a un Cátulo (Castillo), un Virgilio (Expósito) y dos Homeros (Manzi y Expósito, hermano del Virgilio nombrado). En ocasiones, el origen era la intromisión de algún letrado, como en el episodio que se nos cuenta en torno a uno de ellos, el peruano Manuel Beingolea (1875-1953):

Para proporcionarle medios de vida y sacarle de su aislamiento, un alcalde municipal de Barranco le nombró jefe del Registro Civil de la localidad. Mientras en ello estuvo Beingolea hubo pocas inscripciones con nombres comunes. Llegaba un padre a inscribir el nacimiento de su vástago. “¿Qué nombre le va a dar?" preguntaba el cuentista convertido en funcionario. “José, señor”, contestaba el padre. "Hombre, ¡qué ocurrencia! ¡Feo nombre! No, señor, no. Vamos a ponerle Polidoro, que es un lindo nombre, clásico, mitológico". Y sin admitir observación escribía Polidoro. Así creció la fauna de Olimpias, Floras, Eunices, Diodoros, Polux, Fidelios, Clorindas, Onfalias entre la población modesta de Barranco. ${ }^{23}$

Sin una intervención semejante, en el Golfo de México hubo un florecimiento parecido. En una de sus crónicas, dedicada a "nombres raros", el poeta Amado Nervo comenta acerca de la ciudad de San Juan Bautista, hoy Villahermosa, en Tabasco:

\footnotetext{
${ }^{21}$ Guzmán 1979, pp. 27, 275 y 278.

22 López 2001, p. 237.

${ }^{23}$ Sánchez 1951, p. 351.
} 
San Juan Bautista, salvo el calor y las moscas, se parece como una gota de agua a otra gota a los Campos Elíseos. Es una especie de Panteón donde se codean amigablemente todos los semidioses que en el mundo han sido. Las familias tabasqueñas, rindiendo un culto clásico a la antigüedad, culto que las honra sobremanera, en vez de buscar en el calendario nombres para sus recién nacidos, los buscan en la Ilíada, en la Eneida, en la Biblia y en general en la historia universal. Y así en una familia, pongo por caso, hay un Homero, una Cornelia, un Bruto, un Salmanasar y una Hera. En el hogar y la calle son frecuentes diálogos como éstos [...]

- ¿Sabes? Lucio Cornelio Scila anda haciéndole el oso a Semíramis.

- ¿Sí? Pues que lo sepa Kambises y verás cómo les va. ${ }^{24}$

Años después, el mismo rasgo aparecería en las memorias de un nativo de ese estado, el escritor Andrés Iduarte (1907-1984):

Un tabasqueño me decía alguna vez que Tabasco es un país con nombres griegos y alma africana [...] En mi tierra casi todos, jacobinos cuanto positivistas, ponían a los hijos los nombres más extraños. Algunos entraban a saco en la filosofía: en Tabasco abundan los Sócrates, los Platones, los Aristóteles... Otros papás recurrían a la literatura. En una serenata - canciones y guitarras a media noche, al pie de una ventana tras de la cual se supone que escucha la pálida doncella - se reunieron en una ocasión los nombres de los tres trágicos griegos: Sófocles Pérez, Esquilo Ramírez y Eurípides Guardiola $[\ldots]$ a pesar de todas las variaciones y todos los caprichos, los nombres clásicos siguen siendo los preferidos: Píndaros, Alcibíades, Epaminondas, Pericles, Eratóstenes, Arquímedes. Esta rememoración de la cultura [...] no impedía que a los veinte años, por el amor de Aspasia o de Medea, o por celos de Andrómeda o Lysístrata, Armodio y Aristogitón sacaran la pistola de la blusa y, en singular combate, sucumbieran contentos y sonrientes en el Playón o en la Pigua. ${ }^{25}$

\section{Lo que podía saberse}

Dada la lejanía de los referentes, la chanza popular debía cebarse en ellos. ¿Cómo no reírse de grupos que se autonombraban Apolónidas o Pánidas,

${ }^{24}$ Nervo 1962, pp. 619-620.

${ }^{25}$ Iduarte 1951, pp. 108-109. Sobre el tema de los nombres tabasqueños, me sirvió de guía hacia los textos de Nervo e Iduarte la nota de René López 2006. 
de criollos carnudos embutidos en su traje negro y cuello duro que se denominaban entre sí Euforión y Rodión, como hacían los jóvenes porfirianos que eran miembros del Ateneo de la Juventud en México? La picardía fingía confusiones: "Si un Bruto salvó a Roma, ¿cómo diablos / no salvan esta patria tantos brutos?". ${ }^{26}$ O era ironía que subrayaba las absurdidades de la pompa oficial, como en el pueblo de Cuévano, que retrata Jorge Ibargüengoitia, cuyos habitantes se ufanan de que "modestia aparte, somos la Atenas de por aquí", y cuya universidad está dominada por unos viejos profesores llamados los Siete Sabios de Grecia, "por no ser ni siete ni sabios ni griegos". ${ }^{27}$ La comparación de Atenas con villorrios malolientes a veces era demasiado audaz como para no suscitar la ironía: "en esta Atenas de América sólo encienden siete faroles en memoria y reverencia de los Siete Sabios de Grecia”, habría exclamado un español ante la escasa iluminación de Bogotá en $1861 .{ }^{28}$ Otras menciones al carácter ateniense de Bogotá son también ligeramente irónicas.

Pero además, como puede verse en el caso de la onomástica y de los discursos de la Revolución mexicana, el pueblo recicló y seleccionó del repertorio que se le proponía, o reinterpretó los símbolos: en el escudo de Bolivia figura un hacha de los lictores con fasces, aunque la interpretación corriente hace justicia y ve allá un hacha incaica. ${ }^{29}$ Las denominaciones mismas dadas a la estatuaria y el uso que de ellas se hace son más ejemplos: la Victoria de Samotracia que adorna el centro de México se ha convertido en El Ángel, y sirve para concentraciones y actos "futboleros", como también la Minerva tapatía; la otra Minerva, emblema de la Universidad de Buenos Aires, fue siempre para sus alumnos La Gorda. Una burlesca paremiología pseudolatina esmalta la conversación plebeya, y un panteón de dioses y semidioses nacidos del genio popular se erigen en casas particulares y en cementerios provinciales. Las artesanías se apropian de figuras clásicas como las sirenas, presentes en las estatuillas zapotecas de barro negro.

Hay quizá en todo ello una aprehensión del mundo clásico más certera que la de las elites criollas que tuercen el gesto. La formación de éstas no siempre era lo que pregonaban: nos refiere Juan Montalvo de un

\footnotetext{
${ }^{26}$ Composición de 1870 en Palma 2000, pp. 389-390 y 488.

${ }^{27}$ Ibargüengoitia 1975, pp. 1 y 10.

${ }^{28}$ Cordovez Moure 1962, p. 1352.

${ }^{29}$ Burucúa y Campagne 2003, p. 437.
} 
examen en la Universidad de Quito, último requisito para alcanzar un doctorado en jurisprudencia, en el que le preguntaron al examinado las figuras de dicción: "Metempsícosis, parálisis, metamorfosis, parásitos, fósiles, temístocles, hipertobones, respondió con admirable desparpajo el señor doctor", que creemos fue aprobado. ${ }^{30}$ Será la pluma maldita de Montalvo, dirán algunos, pero si vemos de cerca las realidades de la enseñanza, no nos extrañe que nos haya referido una anécdota cierta: en Chile, los educandos se esmeraban en adivinar el orden en que el alemán Federico Hanssen hacía traducir un fragmento de Cornelio Nepote, y cada uno estudiaba exclusivamente ese fragmento; Miguel Cané nos relata en Juvenilia (1882) la suficiencia con que, absolutamente ignorantes del griego, sus compañeros del Colegio Nacional de Buenos Aires valoraban la pronunciación del profesor Larsen, considerada triste ejemplo del dialecto usado por los porteros de Atenas. Alfonso Reyes atestigua cómo el latín y el griego, por exigencias del programa "desaparecían entre un cubileteo de raíces elementales", expuestas a una desordenada turba de muchachos por el plácido profesor Francisco Rivas. ${ }^{31}$

¿Quién, por otra parte, iba a enseñar? Abundaban los aventureros, como aquel gibraltareño que se presentó en una escuela de Buenos Aires pretendiendo dominar inglés, francés y griego. "iQué griego ni que historia, debe ser algún trapalón”, exclamó el director, que le habló en vasco y el farsante fingió entender que era purísimo ático. ${ }^{32}$ Cierta Memoria del Ministerio de Instrucción Pública venezolano, de 1913, confirma cómo "algunos claman contra el latín y el griego, sin contar que su estudio es poco menos que un simulacro en la generalidad de los casos"; un "Viva la Pepa" en realidad: había una cátedra de mitología griega en el Colegio Diez (fundado en 1906), que leía el director, basándose en unos apuntes. ${ }^{33}$ Se cuenta de un maestro originario de Corrientes que enseñaba guaraní a sus alumnos, pero juraba que era el latín que las autoridades educativas argentinas obligaban a impartir.

Y no es aquí, como en otros casos, que las pobres condiciones de nuestros países hayan impedido el avance de la ciencia. Manuales siempre hubo, figuran en los catálogos de las bibliotecas; por otro lado,

\footnotetext{
${ }^{30}$ Montalvo 1977, p. 331.

${ }^{31}$ Hanisch Espíndola 1991, p. 146; Cané 1960, p. 76; Reyes 1960, p. 190.

32 Mansilla 2006, pp. 128, 162.

${ }^{33}$ Fernández 1968, pp. 23 y 25.
} 
muchos de nuestros sabihondos residieron por largas temporadas en Europa, donde no faltaban cátedras, diccionarios, antologías, concordancias, Pauly Wissowas, ruinas ni epígrafes. Pero el facilismo criollo no congenia con las arduas jornadas dedicadas a reconocer un dativo o un ablativo absoluto. Iban a lo fácil, escuchaban conferencias en la Sorbona, conversaban con los que sí sabían, compraban volúmenes ricamente ilustrados.

El argentino que va a Roma, salvo que no sea inteligente, se dedica al oficio de admirador. Tan bien los deben conocer allá a estos bicharracos, que en cuanto los aborígenes caen por esas tierras, los reciben una brigada de lazzaroni y de cicerones, prontos a mostrarle al hombre del cocotero todas las maravillas arqueológicas que encierra esa tierra de tenorios, mandolinistas y peluqueros. Los salvajes quedan locos de admiración, empiezan a escribir sobre el Foro Romano, sobre la arquitectura jónica y dórica, sobre los arquitrabes y otras mil macanas que sólo pueden interesar a un albañil o a un reblandecido. ${ }^{34}$

Es que la escasez de ciencia no obstaba para el despliegue, como el de aquellos jóvenes, "hinchados como odres de vino, sólo porque Calandrelli les untó un poco de griego como quien restriega un diente de ajo sobre rebanada de pan". ${ }^{35}$ A mansalva citaban los "grecolatinos" colombianos, llamados también "grecoquimbayas", cuyo movimiento "tomaba de Grecia y Roma a cuentagotas, pues su espectacular retórica se nutría no tanto de Demóstenes o de Cicerón, cuanto de la poesía española de fines del siglo XIX, aunque también de la poesía metafísica" ${ }^{36}$ Se presentaban dos insignes eruditos criollos como traductores de Homero, uno desde Cuernavaca afirmaba no saber una palabra de griego; el otro, en opinión de su coterráneo Macedonio Fernández, sospechaba dicha lengua (y todavía hay quien les cree). El cantor de nuestra herencia helena, José Enrique Rodó, no sabía ni reconocer un aoristo, y dejó más de una pifia clásica en sus escritos.

Éstas por lo demás abundan: el latín de Atenas antes aludido es una,

${ }^{34}$ Arlt 1975, pp. 73 ss.

${ }^{35}$ Gálvez 1990, p. 120. Se refiere a Matías Calandrelli, alias "El Sabio Calandrelli”, napolitano que, con su arete en la oreja, desplegaba una ciencia inconmensurable en la Argentina de Sarmiento.

${ }^{36}$ Betancur 2002, p. 223. 
y excusable, otras lo son menos. Un diario santiaguino recordaba al público de criollos que no entendían el significado del Habeas corpus y lo relacionaban con la fiesta del Corpus y las habas. ${ }^{37}$ Cierto tribuno venezolano que rememoraba a un Julio César que cruzaba en barco el Rubicón, la conminación de Juan Domingo Perón a realizar "todo en su medida y armoniosamente, como estaba escrito en los frontispicios de las universidades griegas", y el anuncio de su sucesor Carlos Saúl Menem de que solía leer las obras completas de Sócrates. Si nos empeñamos en la Quellenforschung, ya he citado la "latinidad de ediciones Sempere", agrego aquí "algún tratado de divulgación histórica" que sirvió al "Mariscal Greco-Romano", el peruano Domingo Nieto (1803-1844), a exaltar en sus discursos la república romana, o "el grecorromano de manual de Duruy" que sufría el venezolano Rafael Pocaterra. ${ }^{38}$

\section{Conclusiones}

Retomando cosas dichas unos párrafos antes, creo que la apropiación criolla de los clásicos tenía entre sus finalidades la de domesticar a la chusma de indios, negros y castas, al populacho urbano y a las masas enardecidas, asimilándolos a los valores criollos. Zambitopos y zambitopas vocingleros periódicamente andan pidiendo el regreso a una Grecia que no conocen y la operación vuelve a sus practicantes hieráticos e impostados, lo cual, unido a su inseguro manejo de las fuentes y bibliografías en idiomas arcanos, contribuye para que entiendan y traduzcan el mundo clásico en un remedo de la Europa burguesa, moralista y aburrida. El populacho latinoamericano en cambio intuyó siempre que ese mundo debía de parecerse mucho al propio, variopinto, gozador, despreocupado y impertinente. Hasta entenderían mejor que la elite el argumento de Atenea negra.

\section{BIBLIOGRAFÍA}

Arguedas, Alcides, Pueblo enfermo, 3a . ed., La Paz, Puerta del Sol, 1977 [1936].

ARLT, Roberto, Nuevas aguafuertes, Buenos Aires, Losada, 1975.

Besse, Susan K., "Defining a 'national type': Brazilian beauty contests in the

${ }^{37}$ El Duende de Santiago, 29-vi-1818, pp. 89-90.

38 Pocaterra 1979, p. 378. 
1920s", Estudios Interdisciplinarios de América Latina y el Caribe, 16, 1, 2005, s. p. En: <www1.tau.ac.il/eial/index>.

Betancur, Belisario, "El humanista sonreído: Otto Morales Benítez", Cuadernos Americanos, 91, 2002, pp. 221-231.

Burucúa, José Emilio y Fabián Alejandro CAmPagne, "Mitos y simbologías nacionales en los países del Cono Sur", en Antonio Annino y François-Xavier Guerra, Inventando la nación: Iberoamérica siglo XIX, México, FCE, 2003, pp. 433-474.

CANÉ, Miguel, Juvenilia, Buenos Aires: Eudeba, 1960 [1882].

Colección de antiguos periódicos chilenos, Guillermo Feliú Cruz (ed.), Santiago de Chile, Biblioteca Nacional, 1952.

Cordovez Moure, José María, Reminiscencias de Santafé de Bogotá, Madrid, Aguilar, 1962 [1893].

Demasi, Carlos, “La construcción de un 'héroe máximo': José Artigas en las conmemoraciones uruguayas de 1910", Revista Iberoamericana, 71, 2005, pp. 10291045.

Devoto, Fernando J., "Las dos ciudades de Juan Agustín García: de la "ciudad indiana' a la metrópolis del centenario", Estudios Sociales (Santa Fe), 22-23, 2002, pp. 75-93.

El Duende de Santiago, 29-vi-1818, en Colección de antiguos periódicos chilenos, vol. 12, Guillermo Feliz Cruz (ed.), Santiago de Chile, Biblioteca Nacional, 1952, pp. 89-90

FERNÁNDEZ H., Rafael, La enseñanza del griego en Venezuela, Caracas, Universidad Central de Venezuela, 1968.

FORNET, Ambrosio, "El experimento neocolonial cubano y sus repercusiones en el campo intelectual (1898-1923)", en Leopoldo Zea y María Teresa Miaja (comps.), 98: derrota pírrica, México, IPGH/FCE, 2000, pp. 191-209.

GÁLVEZ, Víctor (Vicente G. Quesada), Memorias de un viejo: escenas de costumbres de la República Argentina, est. prel. de Antonio Pagés Larraya, Buenos Aires, Academia Argentina de Letras, 1990 [1888].

GuERRERO, Andrés, "The construction of a ventriloquist's image: liberal discourse and the 'Miserable Indian Race' in late $19^{\text {th }}$ century Ecuador", Journal of Latin American Studies, 29, 1997, pp. 555-590.

Gallegos, Rómulo, Canaima, edición crítica, Charles Minguet (coord.), México, Consejo Nacional para la Cultura y las Artes (Colección Archivos), 1993 [1935].

GuZMÁN, Martín Luis, El águila y la serpiente, prólogo de María del Carmen Millán, México, Promesa, 1979 [1928].

GutiéRrez EstéVez, Manuel, "El amor a la patria y a la tribu: las retóricas de la memoria incómoda", Revista de Antropologia (São Paulo), 47, 2004, pp. 345-377. En: <http://www.scielo.br/scielo.php?script=sci_arttext\&pid=S0034$77012004000200001 \& \operatorname{lng}=\mathrm{en} \& \mathrm{nrm}=\mathrm{iso}>$.

Hanisch Espíndola, Walter, El latín en Chile, Santiago, Biblioteca Nacional, 1991. 
IbARGÜENGOITIA, Jorge, Estas ruinas que ves, México, Joaquin Mortiz, 1975.

IdUARTE, Andrés, Un niño en la Revolución mexicana, México, Ruta, 1951.

KILAPÁn, Lonko, El origen griego de los araucanos, Santiago, Editorial Universitaria, 1974.

Lima Barreto, Dos novelas: Recuerdos del escribiente Isaías Caminha / El triste fin de Policarpo Quaresma, prólogo y cronología Francisco de Assis Barbosa, Caracas, Biblioteca Ayacucho, 1978.

LóPEz, René Alberto, "Nombres raros como Hipotenusa, Belú y Hitler abundan en Tabasco: investigador", La Jornada, 6 de febrero de 2006.

LóPEZ, Vicente F., La novia del hereje o La Inquisición de Lima, Buenos Aires, Emecé, 2001 [1854].

Mansilla, Lucio Victorio, Mis memorias: infancia, adolescencia, Buenos Aires: CM, 2006 [1904].

Menéndez Pelayo, Marcelino, Historia de la poesía Hispano-Americana, Madrid, Librería general de Victoriano Suárez, 1911.

Montalvo, Juan, Las Catilinarias, selección y prólogo Benjamín Carrión, cronología y notas Gustavo Alfredo Jácome, Caracas, Biblioteca Ayacucho, 1977.

Nervo, Amado, "Los Campos Elíseos en Tabasco", crónica del 25 de junio de 1896, en sus Obras completas, tomo I, ed., intr. y notas de Francisco González Guerrero (prosa) y Alfonso Méndez Plancarte (poesías), Madrid, Aguilar, 1962, pp. 619-620.

Osorio Romero, Ignacio, "El helenismo en México: de Trento a los filólogos sensualistas", Nova Tellus, 4, 1986, pp. 63-117.

Palma, Ricardo, Obra poética, comp. de Merlin Compton, Lima, Biblioteca Nacional del Perú, 2000.

Payró, Roberto J., Pago Chico y Nuevos cuentos de Pago Chico, Buenos Aires, Losada, 1969.

PiCón SAlas, Mariano, "Geografía con algunas gentes", en Suma de Venezuela, introducción de Guillermo Sucre, notas y variantes de Cristian Álvarez, Caracas, Monte Ávila (Biblioteca Mariano Picón-Salas, II), 1987 [1949].

PoCATERrA, José Rafael, Memorias de un venezolano de la decadencia, tomo II, La vergüenza de América, 1919-1922, La oposición. Apéndice documental, 19201935, Caracas, Monte Ávila, 1979.

Reclus, Elisée, "Voyage a la Nouvelle Grenade", Revue des Deux Mondes, 24, 1859, pp. 624-661.

REYes, Alfonso, Pasado inmediato, en Obras completas de Alfonso Reyes, vol. 12, México, FCE, 1960.

SÁNCHEZ, Luis Alberto, Balance y liquidación del Novecientos: ¿tuvimos maestros en nuestra América?, 3a . ed. corregida, Lima, Universidad Nacional Mayor de San Marcos, 1968.

__, La literatura peruana: derrotero para una historia intelectual del Perú, tomo 6, Naturalistas, ideólogos y modernistas, Asunción del Paraguay, Guarania, 
1951.

Soto Posada, Gonzalo, "Latín y cultura en Colombia: rastreo a través de la lengua latina de la presencia de la cultura española en la historia colombiana", en Ildefonso Murillo (ed.), El pensamiento hispánico en América, siglos XVI-XX, Salamanca, Universidad Pontificia, 2007, pp. 211-236.

STEPAN, Nancy Leys, "The hour of eugenics": race, gender, and nation in Latin America, Ithaca / London, Cornell University Press, 1991.

URBINA GaITÁn, Chester, "Antiimperialismo y reafirmación nacional: los actos de inauguración del Monumento a Juan Rafael Mora Porras (1929)", Diálogos. Revista Electrónica de Historia, 1, 4, junio-septiembre de 2000, s. p. En: <www.historia.fis.ucr.ac.cr/articulos/1999/vol1-n4/chesterurb.pdf>.

VALLE, Rafael Heliodoro, "Pretérito perfecto", Cuadernos americanos, 64.4, 1952, pp. 252-282.

VILANOVA, Ángel, "La tradición clásica grecolatina y las literaturas venezolana y colombiana de la primera mitad del siglo XIX", Praesentia, 2-3, 1998-1999, pp. 253-283. 